\title{
The unusual UV continuum of quasar Ton 34 and the possibility of crystalline dust absorption
}

\author{
L. Binette $\mathrm{e}^{1,2}$ and Y. Krongold ${ }^{1}$
}

\author{
1 Instituto de Astronomía, UNAM, Ap. 70-264, 04510 México, DF, México \\ 2 Département de Physique, de Génie Physique et d'Optique, Université Laval, Québec, QC, G1K 7P4, Canada
}

Received 27 June 2007/ Accepted 22 November 2007

\begin{abstract}
Context. Luminous quasars are known to display a sharp steepening of the continuum near $1100 \AA$. This spectral feature is not well fitted by current accretion disk models, unless Comptonization of the disk emission is invoked. Absorption by carbon crystalline dust has been proposed to account for this feature.

Aims. Ton $34\left(z_{q}=1.928\right)$ exhibits the steepest far-UV decline $\left(F_{v} \propto v^{-5.3}\right)$ among the 183 quasar HST-FOS spectra analyzed by Telfer et al. (2002, ApJ, 565, 773). It is an ideal object to test the crystalline dust hypothesis as well as alternative interpretations of the UV break.

Methods. We reconstruct the UV spectral energy distribution of Ton 34 by combining HST, IUE and Palomar spectra.

Results. The far-UV continuum shows a very deep continuum trough, which is bounded by a steep far-UV rise. We fit the trough assuming nanodiamond dust grains.

Conclusions. Extinction by carbon crystalline dust reproduces the deep absorption trough of Ton 34 reasonably well, but not the observed steep rise in the extreme UV. We also study the possibility of an intrinsic continuum rollover. The dust might be part of a high velocity outflow $\left(\simeq 13000 \mathrm{~km} \mathrm{~s}^{-1}\right)$, which is observed in absorption in the lines of C IV $\lambda 1549, \mathrm{O}$ VI $\lambda 1035, \mathrm{~N}$ V $\lambda 1240$ and Ly $\alpha$.
\end{abstract}

Key words. ISM: dust, extinction - galaxies: quasars: general - quasars: individual: Ton 34 - ultraviolet: general

\section{Introduction}

The spectral energy distribution (SED) of quasar and Seyfert I galaxies is composed of emission lines superimposed on various continuum emission components. The near-infrared to visible domain is reasonably well reproduced by a powerlaw. The ultraviolet (UV) spectral region is characterized by a broad continuum excess, which is referred to as the big blue bump (BBB). According to general belief, it corresponds to the thermal signature from a hot accretion disk orbiting a supermassive black hole. The extension in the extreme UV of the BBB is expected to provide the ionizing flux that powers most of the emission lines. A serious problem with this picture, however, is that the BBB appears to be too soft $^{1}$ to account for the high excitation lines from the broad emission line region (BELR) (Korista et al. 1997). In effect, a marked continuum decline (i.e. steepening) takes place shortward of $\simeq 1100 \AA$ (rest-frame), which we hereafter refer to as the far-UV break ${ }^{2}$. A possibility might be that this break is more akin to a localized continuum trough, followed by a marked recovery in the extreme UV, which is the energy region responsible for producing the high-excitation emission lines. Various mechanisms that could generate such a trough are summarized by Binette et al. (2007).

Our aim is to probe the nature of the far-UV break and to test alternative interpretations of it by focussing on the more

\footnotetext{
1 There is an apparent inconsistency between the SEDs typically observed and the much harder ones preferred in photoionization BELR models (e.g. Baldwin et al. 1995; Casebeer et al. 2006).

${ }^{2}$ We will also refer to the wavelength domain longward and shortward of the SED steepening (or break) as the near- and far-UV regions, respectively.
}

extreme cases. For instance, many quasar spectra reveal an SED significantly steeper than the "average" SED derived by Telfer et al. (2002, hereafter TZ02), which behaves as $v^{-1.76}$ (in $F_{v}$ ) shortward of the break. Among the 77 far-UV indices measured by TZ02, there were 3 objects with an ionizing continuum steeper than $v^{-3}$. In this respect, Ton 34 (alternatively named PG $1017+280$ or $\mathrm{J} 1019+2745)$ at redshift $z_{q}=1.928$ is the most extreme case, with a powerlaw index as steep as $v^{-5.3}$ (TZ02). For this reason, we consider it an ideal object to test competing models of the physical origin of the $1100 \AA$ break. In this paper, we used different bibliographical sources to build an SED of Ton 34 that is as complete as possible. As the data are of limited quality, we intend to obtain higher quality data that would cover the X-ray domain down to the optical UV.

Our initial objective was to verify whether the hypothesis of absorption by crystalline carbon dust grains of Binette et al. (2005, hereafter B05) would survive the test of modeling the extreme SED of Ton 34. Using two flavors of nanodiamonds, B05 could successfully reproduce the position and detailed shape of the far-UV break in 50 quasars, out of a total sample of 61 objects from HST-FOS archives ${ }^{3}$. More recently, Haro-Corzo et al. (2007, hereafter H07) reduced the dust model to a single flavor (which excludes the meteoritic type), since a particular emission feature expected near $3.5 \mu \mathrm{m}$ with the meteoritic case is absent from the mid-IR spectrum of 3C 298 (de Diego et al. 2007).

\footnotetext{
3 The analysis of B05 included only multigrating HST spectra that extended down to at least $900 \AA$ (rest-frame), i.e. 61 quasars in total. Ton 34 was not included, since only a single grating HST spectrum exists.
} 


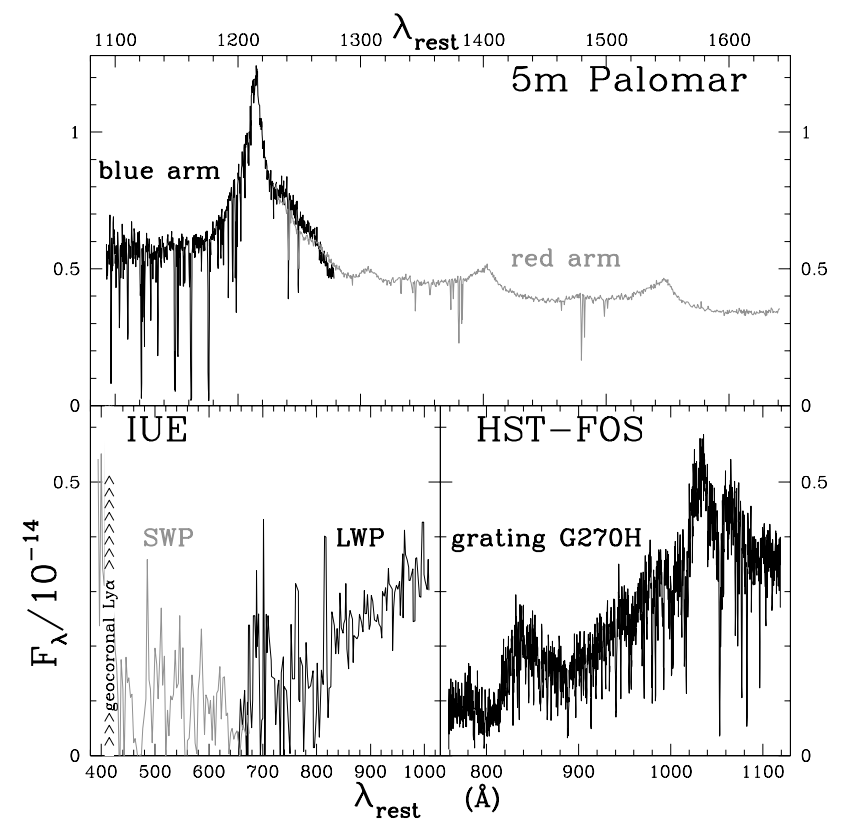

Fig. 1. Spectral segments of Ton 34 extracted from different archival or bibliographical sources. Top panel: blue and red optical spectra (in arbitrary units) from Sargent et al. (1988); lower right panel: HST-FOS spectrum using grating $\mathrm{G} 270 \mathrm{H}$; lower left panel: IUE spectra (LWP and SWP) (see Sect. 2.1).

In this paper, we discuss the merits of the dust absorption model as well as its limitations in reproducing the deep trough that characterizes the Ton 34 SED. In Appendix A, we compare different extinction curves and illustrate how the far-UV extinction is affected. In a companion paper (Binette \& Krongold 2008), we report on some of the peculiarities of the emission line spectrum of this unusual quasar.

\section{The observed UV energy distribution of Ton 34}

\subsection{Description of the archival data}

The current work is based on the following archival or bibliographical sources. The 760-1120 $\mathrm{A}$ spectral segment is provided by the dataset Y2IE0A0AT from the HST-FOS archives. It corresponds to an integration time of $1100 \mathrm{~s}$ (23rd of February 1995) using grating G270H (see lower right panel in Fig. 1). To cover the extreme UV, we then borrowed from the IUE archives. The long wavelength segment (LWP in Fig. 1) is from Tripp et al. (1994) and corresponds to the dataset LW0P5708 (25800 s on 18th of April 1986). Fluxes longward of $3000 \AA$ (observerframe) were severely affected from reflected sunlight or moonlight (Lanzetta et al. 1993) and have been discarded. The shorter wavelength IUE segment (SWP in Fig. 1) was extracted directly from the archives and corresponds to the dataset SWP28188 (23 400s on 4th of October 1985). Because of the limited S/N and to avoid line clutter, the SWP segment shown in Fig. 1 has been re-binned by merging pixels in groups of 5 . The region corresponding to the strong geocoronal Ly $\alpha$ line has been masked.

In order to constrain the SED behavior longward of the HST segment, we adopted the published optical spectra of Sargent et al. (1988), which were taken at the Palomar 5.08 m Hale Telescope (in November 1981 and February 1982). Both optical spectra (blue and red arm segments in top panel of Fig. 1) lacked absolute flux calibration. The authors observed standard stars, which allowed them to provide a relative calibration.

\subsection{Derivation of the UV SED}

The UV SED from Ton 34 was derived in the following manner: we statistically corrected the UV spectral segments for the cumulated absorption caused by unresolved Ly $\alpha$ forest lines, which are responsible for the so-called far-UV "Lyman valley" (Møller \& Jakobsen 1990). For that purpose, we adopted the mean $^{4}$ transmission function for $z_{q}=2$ published by Zheng et al. (1997). We also applied a Galactic reddening correction assuming the Cardelli et al. (1989) extinction curve corresponding to $R_{V}=3.1$ and $E_{B-V}=0.13$. The latter value corresponds to the mean extinction inferred from the $100 \mu$ maps of Schlegel et al. (1998) near Ton 34. The blue and red arm segments have been scaled to overlap smoothly with the HST-FOS segment.

Both the LWP and SWP segments were multiplied by a factor 0.75 . This scaling was necessary so that the LWP segment superimposes the HST-FOS spectrum as closely as possible. Continuum variability is a possible explanation for this continuum difference, since the IUE and HST observations were made in different years.

To derive the UV SED shown in Fig. 2, all the spectral segments were shifted to rest-frame wavelengths, and $F_{\lambda}$ was multiplied by $1+z_{q}$. The IUE spectra have been re-binned by grouping $n$ pixels together (SWP with $n=5$ and LWP with $n=3$ ) in order to improve the limited $\mathrm{S} / \mathrm{N}$ and to avoid overcrowding due to line cluttering. The different spectral segments have been color coded as follows: SWP, red; LWP, orange; HST-FOS, blue; and Palomar, dark green.

\subsection{Originality and limitations of the data}

The far-UV HST segment is characterized by a very sharp drop. The spectral index is as steep as -5.3 according to TZ02. We emphasize that both the LWP and the HST data confirm the existence of a sharp flux decline. The SED as a whole suggests the existence of a very deep trough, which reaches its lowest point at $\sim 650 \AA$, followed by a flux rise shortward of the Ly $\alpha$ geocoronal line, as indicated by the SWP segment.

How can we explain the existence of such a deep trough in the far-UV? If it was due to the Lyman Valley, it would imply an increase of a factor of more than 5 in the density of $\operatorname{Ly} \alpha$ forest absorbers. This excess would have to extend along the line of sight over a redshift span of order unity, which appears to be very unlikely. The existence of a few thick absorbers is another possibility. However, such absorbers would result in sharp saturated absorption lines, unlike the progressive drop in flux observed in both the LWT and the HST spectra.

In our opinion, the deep trough seen in Ton 34 is a manifestation of the far-UV break commonly observed in quasars, albeit in a more extreme form. If the steep flux rise seen in Ton 34 towards $400 \AA$ was confirmed, it would lend support to the absorption hypothesis presented in Sect. 3. Alternative explanations could also be explored (cf. Binette et al. 2007).

4 This correction is statistical in nature, as it relies on the average behavior with redshift of the spatial density of intervening absorbers. It cannot be used to correct small portions of the continuum, which may be coincident with a "clear patch" or an over-density in the Ly $\alpha$ forest. These inhomogeneities may generate spurious narrow features, which should not be attributed to emission lines. 


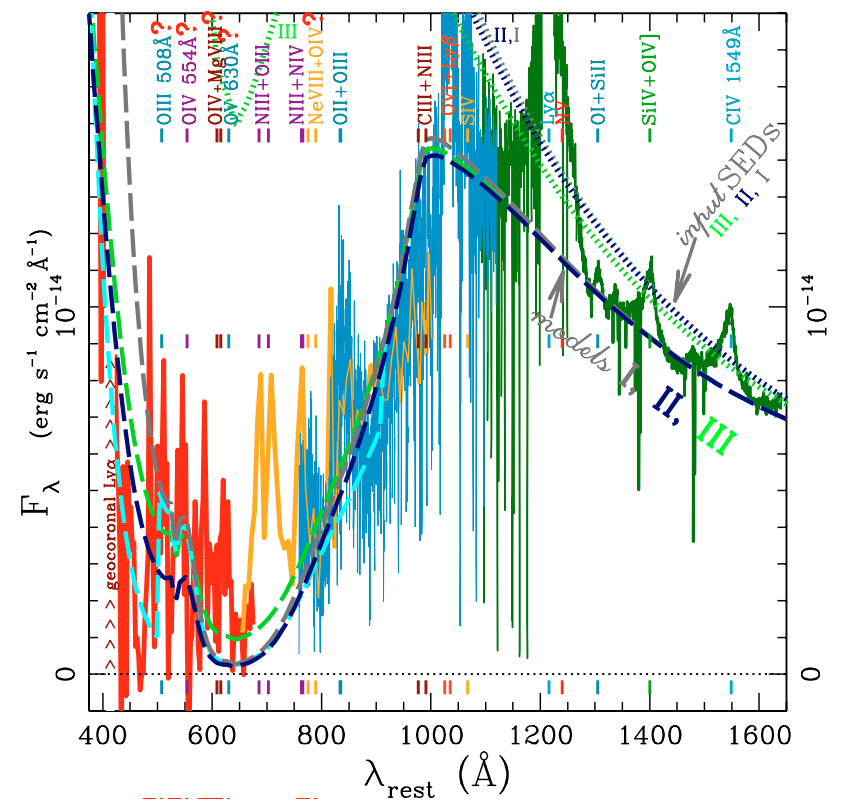

Fig. 2. The UV SED in $F_{\lambda}\left(\mathrm{erg} \mathrm{cm}^{-2} \mathrm{~s}^{-1} \AA^{-1}\right)$ of Ton $34\left(z_{q}=1.928\right)$ as a function of rest-frame wavelength. The near-UV spectrum (dark green continuous line) is from Sargent et al. (1988) and has been scaled to overlap the HST-FOS spectrum (blue line). The far-UV segments correspond to the LWP (orange line) and SWP (red line) spectra from IUE, and have been scaled by a factor 0.75 (see Sect. 2.2). The whole SED has been corrected for Galactic reddening $\left(E_{B-V}=0.13\right)$ and for the cumulative absorption by unresolved intergalactic $\operatorname{Ly} \alpha$ forest lines. The geocoronal Ly $\alpha$ emission line has been masked. Color-coded fiducial marks indicate the position of observed or expected (labeled with symbol “?”) emission lines. Dust absorption Models I, II and III overlay the observed spectrum using color-coded dashed lines. The dotted lines of the corresponding color represent the input SED before absorption (these are reproduced in $\log F_{\lambda}$ in Fig. 3a). Model I (silver line): powerlaw SED absorbed by a slab of column $N_{20}^{\mathrm{H}}=5.3$. Model II (navy blue line): powerlaw SED with a rollover at $670 \AA$ absorbed by a slab of column $N_{20}^{\mathrm{H}}=5.3$. Model III (color lime): same SED, first absorbed by a slab with $N_{20}^{\mathrm{H}}=1.0$ (dotted lime line is SED III), then further absorbed by a dust screen with thickness $N_{20}^{\mathrm{H}}=4.3$ and a leakage of $5 \%$. The cyan colored dashed line is the same as model I, except that it also considers the maximum column allowed for absorption by atomic HI and He I, which is $4 \times 10^{16}$ and $2 \times 10^{17} \mathrm{~cm}^{-2}$, respectively (see Sect. 3.2.4). All four dashed-line absorption models have been normalized to the same flux of $\mathcal{B}=7.3 \times 10^{-15} \mathrm{erg} \mathrm{cm}^{-2} \mathrm{~s}^{-1} \AA^{-1}$ at $1610 \AA$ (see Eq. (1)).

As can be gathered from Fig. 2, the strongest emission features in the far-UV coincide with the position of lines observed or expected in quasar spectra. The strengths of some of these lines are unusual and are the subject of a separate paper (Binette \& Krongold 2008).

\section{Dust extinction models}

\subsection{Carbon crystallite extinction}

TZ02 suggested that dust might play a role in the three quasars that presented the steepest far-UV indices. B05 found that only by considering crystallite carbon grains could they reproduce those SEDs, which had the most pronounced far-UV breaks (the so-called class B spectra in B05), because the extinction in that case is characterized by a relatively sharp threshold in the UV. Since Ton 34 shows the most extreme class B spectrum, we adopt the corresponding extinction curve D1 from B05, which was computed assuming a powerlaw size distribution $\left(a^{-3.5}\right)$ of spherical grains consisting of sizes ranging between 3 and $25 \AA$. The grain composition corresponds to cubic nanodiamonds ${ }^{5}$ (i.e. without surface adsorbates).

A review of the advantages of dust grains consisting of nanodiamonds is presented in Appendix A. We do not consider the nanodiamond hypothesis as the final answer (see Binette et al. 2007), but as one possibility that warrants further study. For the sole purpose of procuring a convenient normalization of the extinction cross section of the dust model labeled D1 (shown in Fig. A.2), all carbon was assumed to be depleted onto dust, with a solar carbon abundance (this assumption is relaxed in Sect. 3.2.5).

The transmitted flux across the dusty material is given by $F_{\lambda}^{\text {obs }}=T_{\lambda} F_{\lambda}^{q}$, where $T_{\lambda}$ is the transmission function, which we approximate with an exponential $\mathrm{e}^{-\tau_{\lambda}^{\text {ext }}}$. Since the albedo for the D1 extinction curve is negligible, scattering need not be considered for the transfer. The opacity is $\tau_{\lambda}^{\text {ext }}=N_{\mathrm{H}} \sigma_{\lambda}^{\mathrm{H}}$, where $\sigma_{\lambda}^{\mathrm{H}}$ is that given by curve $\mathrm{D} 1$ and $N_{\mathrm{H}}$, the $\mathrm{H}$ column density is a free parameter determined by the fitting of the UV trough. Hereafter, we will use $N_{20}^{\mathrm{H}}$, which is the $\mathrm{H}$ column in units of $10^{20} \mathrm{~cm}^{-2}$. As a rule of thumb (and coincidentally), the peak opacity at $640 \AA$ is simply given by $N_{20}^{\mathrm{H}}\left(\simeq \tau_{640}\right)$. We will assume that the intrinsic SED of Ton 34 follows a powerlaw in the near-UV, for which the index $\alpha_{v}$ and normalization constant $\mathcal{B}$ are defined as follows:

$$
F_{\lambda}^{q}=\mathcal{B}\left(\frac{\lambda}{\lambda_{\mathrm{r}}}\right)^{-\left(2+\alpha_{\nu}\right)}
$$

where $\lambda_{r}=1610 \AA$ is used as a reference wavelength. As is customary, the SED indices are defined in the plane $F_{v}\left(\propto v^{+\alpha_{v}}\right)$.

\subsection{Modeling the far-UV continuum, assuming dust absorption}

\subsubsection{The simple powerlaw case}

Longward of the break, the near-UV is well reproduced by using an index $\alpha_{v}=-0.3$. Although small, the extinction within the near-UV domain (for curve D1) cannot be neglected in the case of Ton 34. Therefore, the assumed intrinsic SED must be somewhat harder than observed. We find that an index of +0.1 is favored by our dust models. The dotted line labeled I illustrates such a continuum in both Figs. 2 and 3. To reproduce the trough, a dust screen of column $N_{20}^{\mathrm{H}}=5.3$ is required. The resulting fit is represented by the silver dashed line in Fig. 2. The model presents a reasonable approximation of the trough, but the steep far-UV recovery occurs too early in this Model I, that is, longward of the observed rise.

\subsubsection{The case of a powerlaw with a far-UV roll-over}

There are no X-ray observations reported for Ton 34. The source was not detected by the ROSAT All Sky Survey (RASS). However, we find that the extrapolation of the assumed powerlaw (see Fig. 3) leads to a $0.1-2.4 \mathrm{keV}$ (observer-frame) flux 3-4 orders of magnitude larger than the RASS flux limit of $5 \times 10^{-13} \mathrm{erg} \mathrm{cm}^{-2} \mathrm{~s}^{-1}$ (Voges et al. 1999). Therefore, the nearUV powerlaw must steepen considerably in the far-UV or in the soft X-rays, but we have no direct indication as to where. B05 proposed the existence of a roll-over in the extreme UV, to

5 Instead of using two types of nanodiamonds (cubic and meteoritic) as in B05, H07 could fit the more numerous class A sEDs using only cubic nanodiamonds (without the meteoritic type), but with a much wider grain size distribution, which extended from 3 to $200 \AA$. 


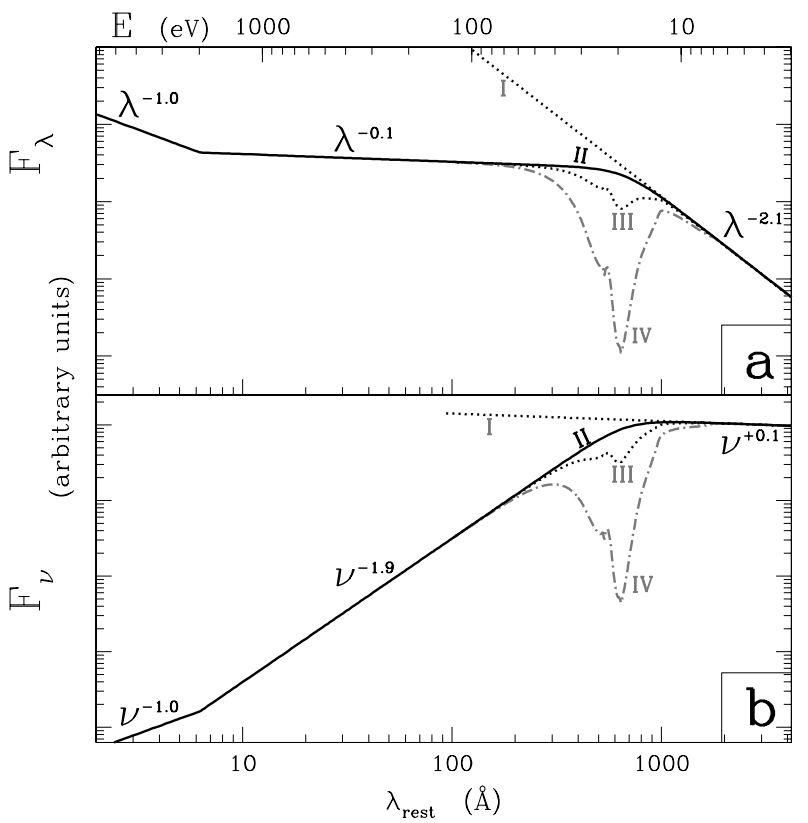

Fig. 3. Log-log plot of the three input SEDS I-III in either $F_{\lambda}$ panel a) or $F_{v}$ panel b) as a function of wavelength (bottom axis) or photon energy (top axis). The transmitted SED assuming $N_{20}^{\mathrm{H}}=5.3$ is shown by the dash-dotted line, labeled IV above.

connect the UV domain with the X-rays. In their study of 11 individual quasar SEDs, H07 included the same roll-over. They parameterized it using the function

$C_{\lambda}=\left(1+\left[\lambda / \lambda_{\mathrm{brk}}\right]^{f \delta}\right)^{-f^{-1}}$

where $\delta(<0)$ is a measure of the steepening, $f$ a form factor and $\lambda_{\text {brk }}$ the wavelength of the cut-off. The values considered by these authors are $f=2.8$ and $\lambda_{\text {brk }}=670 \AA(18.5 \mathrm{eV})$. By multiplying the near-UV powerlaw of Eq. (1) by this function $C_{\lambda}$, a shallow steepening now takes place at $\lambda_{\text {brk }}$, which has the effect of incrementing the powerlaw index by $\delta$ in the far-UV. The value of $\delta$ favored by B05 was -1.6. However, H07 showed that either the index change $\delta$ is significantly larger, or the rollover must take place at significantly higher energies. For Ton 34, we adopted a value of $\delta=-2.0$, justified as follows: the $\alpha_{\mathrm{OX}}$ index, defined by the flux ratio between $2500 \AA$ and $2 \mathrm{keV}$, tends to fall in quasars in the interval -1.5 to -1.6 (Anderson \& Margon 1987; Green et al. 1995; Avni et al. 1995; Yuan et al. 1998). Our chosen value of $\delta=-2.0$ results in an $\alpha_{\mathrm{OX}}=-1.45$, which is consistent with these determinations. It implies an X-ray flux of $\sim 6 \times 10^{-13} \mathrm{erg} \mathrm{cm}^{-2} \mathrm{~s}^{-1}$ between $0.1-2.4 \mathrm{keV}$ (observer-frame), consistent with the RASS limit quoted above.

Such a SED consisting of the powerlaw I multiplied by $C_{\lambda}$ is labeled II and shown in either panel of Fig. 3. For the purpose of calculating photoionization models (Binette \& Krongold 2008), we truncated SED II at $2 \mathrm{keV}$ and appended a harder powerlaw to describe the hard X-ray domain. An index of $\simeq-1.0$ better approximates the typical photon indices $(\sim 2)$ measured in the 2$10 \mathrm{keV}$ domain (e.g. Williams et al. 1992; Lawson et al. 1992).

The dust absorption model assuming SED II is represented by the navy blue dashed line in Fig. 2. It has the same dust screen column, $N_{20}^{\mathrm{H}}=5.3$, as the previous model using SED I. The fit to the steep far-UV rise has improved considerably with respect to the previous Model I.

\subsubsection{The partially leaking case}

We note that the observed trough shows at its lowest point a non-zero flux higher than that of Model II. This flux is not necessarily all continuum. It is possible that resonance lines such as N II at $\lambda 645 \AA$ might be contributing near the bottom of the trough. Assuming that the minimum flux is all continuum, we investigate the possibility of leakage of the screen as a result of small inhomogeneities of the screen or of partial covering of the source. As the opacity increases, the relative importance of leakage increases relative to the transmitted flux, until it eventually dominates when $\tau_{640} \gg 1$. To illustrate this, we have considered a partially covered source and found that a leakage of order $5 \%$ suffices to reproduce the observed minimum flux. This is illustrated by the lime dashed-line Model III, which represents the transmitted+leaked flux from input SED III (see Figs. 2 and 3). This input SED III corresponds to SED II, but absorbed by a column of $N_{20}^{\mathrm{H}}=1.0$ (without any leakage). The intention was to use an input SED that already reproduces the moderate UV break found in the more common class A quasars studied by B05. The outer dust screen is characterized by a column of $N_{20}^{\mathrm{H}}=4.3$. Hence, the total absorption column is the same as in the dust absorbed Model II, but the far-UV flux rise is now better reproduced (Fig. 2).

\subsubsection{Limits on atomic gas absorption}

It may be that the roll-over in quasars takes place at higher energies, as suggested by $\mathrm{H} 07$, and that the offset of the flux rise has a different origin or is the result of the limited $\mathrm{S} / \mathrm{N}$ of the data. One possibility to consider is absorption by atomic gas, since dust and gas must coexist within the screen. We determined the maximum amount of photoelectric absorption by H I and He I that provided an acceptable fit to the UV trough. This is represented by the cyan dashed line in Fig. 2. Note that the input SED I used in this case does not contain any roll-over function $C_{\lambda}$. The maximum columns of atomic gas inferred for this Model I with $N_{20}^{\mathrm{H}}=5.3$, which still leads to an acceptable fit of the UV trough, are $N=4 \times 10^{16} \mathrm{~cm}^{-2}$ and $2 \times 10^{17} \mathrm{~cm}^{-2}$, for $\mathrm{HI}$ and $\mathrm{He} \mathrm{I}$, respectively. The main conclusion is that dust models imply that the associated gas must be highly ionized. The limits on the neutral fraction of either species depends on the dust-to-gas ratio, which can easily be 10 times below that assumed here. On the other hand, the metallicity inferred from quasars can be an order of magnitude higher (Hamann \& Ferland 1999; Dhanda et al. 2007) than solar, which would compensate for the effect of using a lower dust-to-gas ratio. For our assumed dust-to-gas ratio (see Sect. 3.1), we derive an upper limit for the hydrogen neutral fraction of $\leq 8 \times 10^{-5}$. For helium, assuming a relative abundance of $10 \%$, the limit on the neutral fraction is $\leq 4 \times 10^{-3}$.

Both values are quite small, especially in the case of $\mathrm{HI}_{\mathrm{I}}$ from which we infer an ionization parameter $U \simeq 0.05$ (estimated using the code MAPPINGS Ic and SED II). If we were to consider collisional ionization instead of photoionization, both neutral fractions are attained when the temperature is in the range $60000-63000 \mathrm{~K}$. The high ionization of the screen probably implies that the dust is not in equilibrium with the radiation field of Ton 34. We note that photoelectric absorption by He I actually improves the fit to the far-UV rise. Interestingly, the SED from the best studied quasar for absorption line purposes, HE 2347-4342, reveals a significant dip near the He I ionization threshold at $504 \AA$ (Reimers et al. 1998; Binette et al. 2007). 


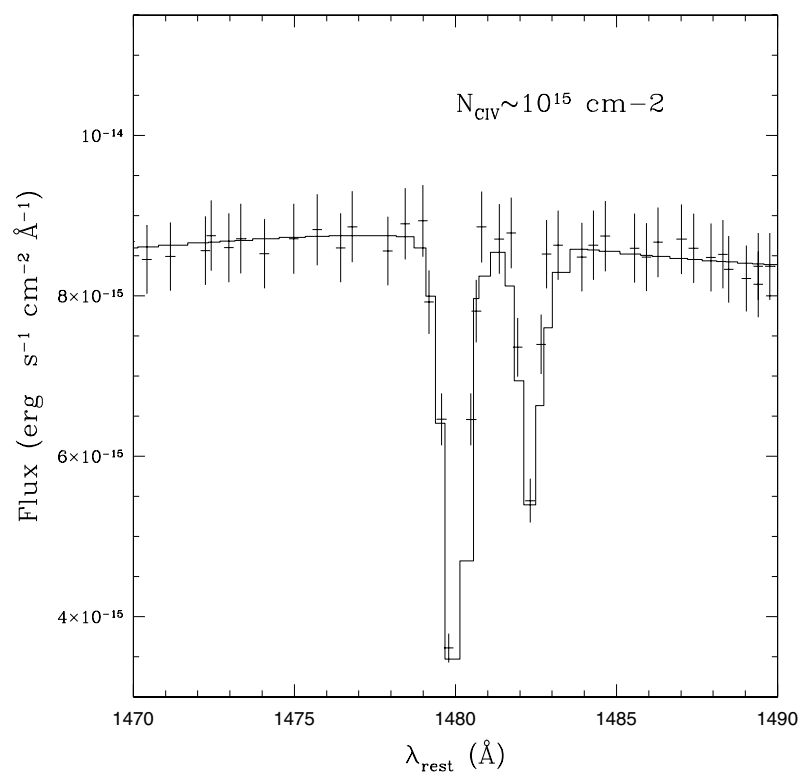

Fig. 4. Plot of the C IV absorption system blueshifted by $13200 \mathrm{~km} \mathrm{~s}^{-1}$. The continuous line is a fit to the C IV doublet assuming a column of $N_{\mathrm{CIV}}=10^{15} \mathrm{~cm}^{-2}$.

\subsubsection{An outflowing ionized absorber?}

The high ionization of the gas required by the dust model (see Sect. 3.2.4) leads us to expect the presence of absorption lines from highly ionized species. In particular, the C IV $\lambda 1549$ line should be present at some level unless all of $\mathrm{C}$ were fully depleted into dust or overly ionized. We have searched for the presence of absorption features in the spectra of Ton 34 . We found a significant C IV absorption system near $1480 \AA$ (see Fig. 4). Assuming that this absorption system is associated with Ton 34 and contains the crystalline dust responsible for the UV trough, we derive an outflow velocity of $13200 \mathrm{~km} \mathrm{~s}^{-1}$, and after fitting the C IV doublet (solid line in Fig. 4), we estimate the column density to be $N_{\mathrm{CIV}} \sim 10^{15} \mathrm{~cm}^{-2}$.

In Sect.3.2.1 we assumed that all of $\mathrm{C}$ was in crystalline form for the sole purpose of procuring a convenient normalization. This resulted in an $\mathrm{H}$ dust screen column $N_{20}^{\mathrm{H}}=5.3$. The total column of $\mathrm{C}$ that need to be "depleted onto dust" in the dust Models I-III presented in Fig. 2 is equivalent to $N_{\text {C.dust }} \sim$ $1.9 \times 10^{17} \mathrm{~cm}^{-2}$. We now explore different scenarios concerning metallicity and depletion that can provide the required dust column.

If we adopt the same photoionization model quoted in Sect. 3.2.4, which is consistent with our estimated limit on $\mathrm{HI}$, we infer $^{6}$ that $13 \%$ of gaseous $\mathrm{C}$ is in the form of $\mathrm{CIV}$ and, therefore, the total column of gaseous $\mathrm{C}$ present is $N_{\mathrm{C} \text {.gas }} \sim$ $7.7 \times 10^{15} \mathrm{~cm}^{-2}$, which is very small by comparison with the amount needed in crystalline form. The total carbon column (gas + dust) becomes $N_{\mathrm{C}} \sim 2.0 \times 10^{17} \mathrm{~cm}^{-2}$, which translates into a metallicity only $3 \%$ higher than solar. The depletion of $\mathrm{C}$ onto dust in this case is $\delta_{\mathrm{C}}=0.96$. A smaller depletion fraction or a higher ionization parameter would both require larger metallicities. For instance, a much smaller depletion fraction of 0.1 and a $\mathrm{C}$ IV/C ratio of 0.13 or 0.013 would imply metallicities of

${ }^{6}$ In this same model, the relative fractions of species $\mathrm{C} \mathrm{V} / \mathrm{C}, \mathrm{N}$ V/N and $\mathrm{O}$ VI/O are $72 \%, 24 \%$ and $14 \%$ respectively.

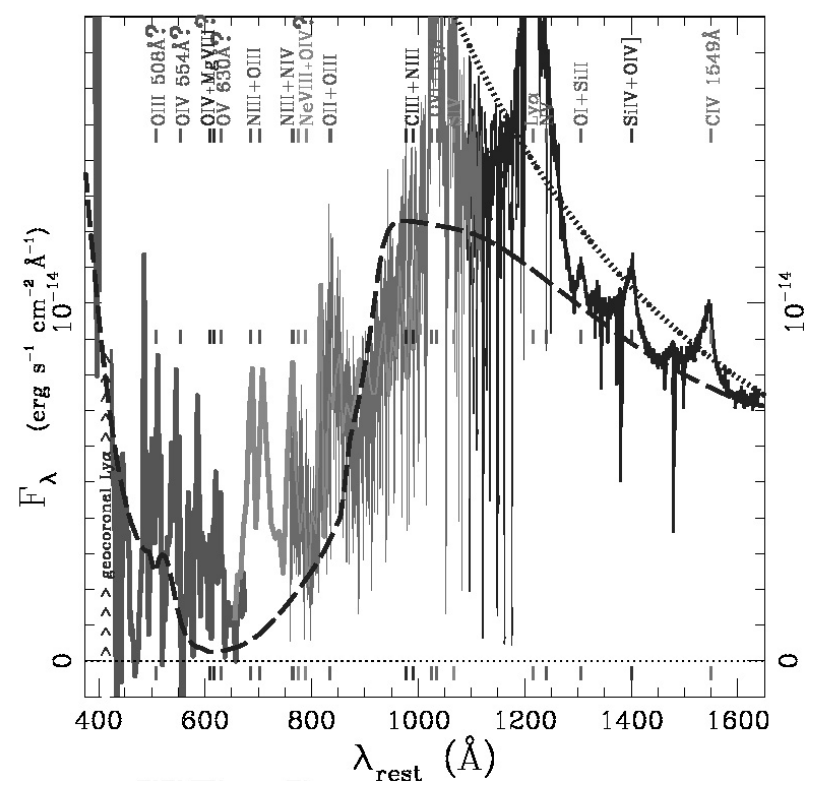

Fig. 5. Same UV energy distribution as in Fig. 2 (grey scale). The dotted line corresponds to SED II and the dashed line represents this SED absorbed by nanodiamond dust $\left(N_{20}^{\mathrm{H}}=5.8\right)$. The dust is assumed to be present in the C IV absorption system that we interpret as outflowing at a velocity of $13200 \mathrm{~km} \mathrm{~s}^{-1}$ (see Sect. 3.2.5 and Fig. 4). The dust grain size distribution in this model extends from 3 to $100 \AA$. Absorption by atomic $\mathrm{HI}$ is also included, assuming the same column as derived in Sect. 3.2 .4 (i.e. $4 \times 10^{16} \mathrm{~cm}^{-2}$ ). Fiducial marks indicate the position of observed or expected (labeled with symbol "?") emission lines.

4.6 and 9 times solar, respectively ${ }^{7}$. We note that the environment of quasars is often characterized by much larger than solar metallicities (e.g. Dhanda et al. 2007; Hamann \& Ferland 1999).

Absorption lines of N V $\lambda 1240$ and O VI $\lambda 1035$ are also observed at the same velocity shift. In the case of O VI, the doublet is severely blended with narrow Ly $\alpha$ lines, which prevents us from estimating its column. As for $\mathrm{N} \mathrm{V}$, the column is estimated at $7 \times 10^{14} \mathrm{~cm}^{-2}$, which is much smaller than that expected from the $U=0.05$ model, assuming solar metallicity. A much higher ionization parameter is thus favored by N v. Finally, a strong Ly $\alpha$ absorption line is also found at the outflow velocity of $13200 \mathrm{~km} \mathrm{~s}^{-1}$. The line is slightly saturated, allowing us only to derive a lower limit to the H I column of $\simeq 2 \times 10^{15} \mathrm{~cm}^{-2}$, consistent with the upper limit on allowed H I in Sect. 3.2.4 of $N_{\mathrm{HI}}=4 \times 10^{16}$. Increasing (decreasing) the ionization parameter would decrease (increase) this upper limit.

When the dust model is shifted in velocity, the fit to the farUV break worsens significantly. It results in an unacceptable flux excess at the onset of the UV trough near $950 \AA$. The dust model can be improved and made to fit both extremities by broadening the grain size distribution, although by half as much as proposed by H07. We show in Fig. 5 our best fit assuming SED II and based on a grain size distribution that extends from 3 to $100 \AA$. The dust screen contains $10 \%$ more dust than previous models (i.e. $N_{\text {C.dust }} \sim 2.1 \times 10^{17} \mathrm{~cm}^{-2}$ ) and the above metallicity estimates must be scaled accordingly. Overall, the fit is quite acceptable.

We note that the outflow velocity of the absorbing material is large compared to that observed in narrow absorption line systems found in Seyfert galaxies (Crenshaw et al. 2003). However, Ton 34 is much brighter than those objects. In addition, this

${ }^{7}$ For a fixed $N_{\text {C.dust }}$, the total column $N_{20}^{\mathrm{H}}$ implied scales inversely with metallicity and with $\delta_{\mathrm{C}}$. 
system seems somewhat strong to be part of the IGM, as the metallicity of the Ly $\alpha$ forest at redshifts similar to that of Ton 34 is at least 2 orders of magnitude smaller than solar (e.g. Pettini 2006). Thus, it is certainly possible that both the ionized absorption lines and the spectral shape in the spectra of Ton 34 are produced by the same screen of material. This would require that the dust is part of a large scale outflow, as suggested in Sect. 4.

\section{Discussion on the crystallite dust model}

The SED observed in Ton 34 is rather unique due to a striking lack of soft ionizing photons. The far-UV break is definitely more extreme than in the other quasars of the TZ02 sample (B05). There is no consensus on the physical origin of the quasar farUV break. Binette et al. (2007) have recently presented a review of alternative interpretations, some of which, however, lack detailed calculations that prevent making a direct comparison with observations. Among the promising interpretations lies the possibility of continuum reprocessing by a wind arising from the accretion disk or absorption from gas progressively accelerated up to quasi relativistic velocities (Eastman et al. 1983). In this paper, we explore the dust absorption interpretation, for which we have a detailed model, but it is certainly premature to exclude other interpretations at this stage.

The nanodiamond dust absorption hypothesis is consistent with the Ton 34 SED, since it produces a broad absorption feature that is compatible with the position, depth and shape of the observed trough. However, the far UV-rise takes place at slightly shorter wavelengths than predicted by the simplest model (Model I). If not due to limitations in the $\mathrm{S} / \mathrm{N}$ or systematics in the instrumental system of IUE, this can be accounted for by assuming a roll-over near $670 \AA$ or photoelectric absorption by He I. Alternatively, the shortcoming might result from the limitations of the dust grain model. Improved quality and higher resolution spectra will be essential to determine the true continuum level at the bottom of the trough and to confirm whether a very steep rise occurs in the extreme UV.

Reproducing the observed continuum trough, in any case, is not sufficient to vindicate the dust model. For instance, we need to find evidence in the mid-IR of re-radiation of the energy absorbed by the dust. Using Spitzer data on 3C 298, de Diego et al. (2007) ruled out the presence of emission bands at 3.43 and $3.53 \mu \mathrm{m}$ as is expected in the case of meteoritic nanodiamonds (i.e. nanodiamonds with surface adsorbates that produce $\mathrm{C}-\mathrm{H}$ stretch emission; Andersen et al. 1998; Jones \& d'Hendecourt 2000). The model used in this paper is not affected, since it is based on cubic diamonds, which may emit either via the onephonon mode due to internal bulk impurities within the crystals (Andersen 1999) or, if made of pure crystals, via multi-phonon modes, which are unfortunately quite inefficient (Braatz et al. 2000; Edwards 1985; Jones \& d'Hendecourt 2000). However, testing these two remaining possibilities still remains to be done.

The absence of strong bound-free absorption of H I shortward of the Lyman limit implies a high ionization parameter ( $U \gtrsim 0.05$ ) for the dust screen, if we assume photoionization (or a temperature above $63000 \mathrm{~K}$ for the thermally ionized case). In such an environment, crystallite dust would not lie in thermal equilibrium with the ambient gas and therefore must continually be replenished at a rate near that at which it is being destroyed. A dust screen within a thin funnel-shaped wind structure, as contemplated for the ionized gas by Elvis (2000) and as inferred from the warm absorbers in NGC 4051 (Krongold et al. 2007), might resolve this problem. Two conditions would have to be met, however: the wind must be launched from a cool region of the accretion disk where the dust can be formed (see Konigl \& Kartje 1994; Everett 2005) and the wind must cross our line-ofsight to the UV emission region of the disk.

The possibility of such a wind is particularly relevant to the problem of detecting the expected absorption lines (such as C IV or O VI) associated with the dust. We do find such lines in an absorption system that is blueshifted by $13200 \mathrm{~km} \mathrm{~s}^{-1}$. To validate this possibility, it is necessary to confirm the presence of similar absorption systems in other quasars that possess a prominent far-UV break.

To conclude, despite the success of the crystalline dust model in reproducing reasonably well the far-UV trough observed in Ton 34 and in other quasars (BI05), there arise various objections to such models that we have partially addressed in this paper. Further studies are needed to reach firm conclusions in favor of or against the possibility of crystalline dust as an explanation of the far-UV break in quasars.

Acknowledgements. This work was supported by the CONACyT grants J-50296 and J-49594, and the UNAM PAPIIT grant IN118905. Diethild Starkmeth helped us with proofreading.

\section{Appendix A: Comparison of different dust models}

\section{A.1. How grey is the UV extinction?}

There have been many interesting discussions in the literature about which extinction curve is the most appropriate for active galactic nuclei (AGN). Following an analysis of 72 optical spectra (of Baker \& Hunstead 1995) of FR II radio-quasars and broad-line radio-galaxies, Gaskell et al. (2004) derived an extinction curve, which is essentially flat ("grey") shortward of $3800 \AA$ A. However, Crenshaw et al. (2001), Cerny et al. (2004), Gaskell \& Benker (2007), Richards et al. (2003) and Willott et al. (2005) found an extinction that is increasing towards the UV, at least down to $1200 \AA$, and maybe beyond. These studies tend to agree that there is little or no evidence of the absorption feature near $2175 \AA$ (see review by Li 2007), a feature that is otherwise striking in the case of Galactic extinction. The presence of a significant amount of scattered light in the particular case of radio-galaxies may be related to the greyness of the extinction, which was inferred by Gaskell et al. (2004) from the Baker \& Hunstead (1995) sample. In their spectro-polarimetric study, Vernet et al. (2001) concluded that the scattering efficiency of quasar light within their sample of high-redshift radio-galaxies is essentially grey. The explanation given is that the condensations that cause the scattering are opaque throughout the UV domain. In this case, the scattering efficiency depends only on the albedo, not on selective extinction. As it turns out, the albedo of Galactic dust is approximately grey except near the $2175 \AA$ feature.

\section{A.2. Multi-component dust models and accretion disks}

Most studies find evidence of an extinction that is rising towards the far-UV in type I AGN (i.e., excluding radio-galaxies). There are no firm conclusions yet about where in the UV the extinction becomes grey and starts to decline. It is likely that the dust grains vary in composition and optical properties according to the particular line-of-sight in which the observer happens to lie. Furthermore, there can be more than one dust component present in any given line of sight. For instance, Gaskell \& Benker (2007) suggest an extinction similar to the Galactic (although without the usual $2175 \AA$ absorption feature), but accompanied, at least in some of their AGN, by additional extinction due to a SMClike dust component. The possible presence of more than one 
dust component is the starting point of our attempt to account for the far-UV break of quasars, for which we will assume it is due to an additional dust component. We used an inverse technique, however, by exploring different dust compositions and size distributions, until the extinction curve we calculated could account for the very sharp break observed near $1100 \AA$.

We emphasize that we are not questioning the general belief that the $\mathrm{BBB}$ corresponds to emission from an accretion disk around a supermassive blackhole. A persistent problem, however, is that the observed BBB is much too soft to account for the high excitation emission-lines typical of quasars (KO97; Koratkar \& Blaes 1999). Our aim has been to explore alternative solutions. Some of these have been ruled out, while others still lack detailed calculation to enable proper testing (see review by Binette et al. 2007). In our opinion, either there exists a secondary ionizing continuum component in the (unobserved) extreme UV (KO97; Binette et al. 1988), or the observed steepening of the UV continuum is a relatively narrow feature followed by a continuum recovery at higher energies (this paper). Since all dust models reach an absorption peak somewhere in the near or far-UV, followed by a decrease in cross-section towards the soft X-rays, the dust hypothesis always implies a continuum recovery towards the extreme UV.

\section{A.3. The far UV-break in Ton 34 and various dust models}

From an early exploration of various dust models B05 concluded that crystalline nanodiamond dust had the required optical properties to reproduce the far-UV break of quasars. The proposed dust model is based on a relatively abundant element, namely carbon. We compare this with alternative dust models below.

In Fig. A.1 we show the extinction curve D1 used in the current paper (black continuous line). For all models shown below, we assume a powerlaw size distribution $\left(\propto a^{-3.5}\right.$, see Mathis et al. 1977; Draine \& Lee 1984). The curve D1 illustrates the small size regime ( $a \leq 25 \AA$ ) for nanodiamonds, while curve D3 is characterized by grain sizes spanning a wider range of $3 \leq a \leq 200 \AA$. The SED steepening in Class B spectra (defined in B05), such as in Ton 34, is well fitted using dust model D1 while the more numerous Class A spectra appear to favor extinction model D3 (see H07). In Fig. A.2, we show a simple powerlaw absorbed by a dust screen of column $N_{20}^{\mathrm{H}}=5.3$ characterized by an extinction given by model D1.

We now compare the extinction resulting from other dust models shown in Fig. A.1. The silver line corresponds to a model of the Galactic extinction as calculated by Martin \& Rouleau (1991). It contains an equal number of graphite and silicate grains with grain sizes encompassing the range $50 \leq a \leq$ $2500 \AA$. A comparison with the Ton 34 is presented in Fig. A.2 assuming a column $N_{20}^{\mathrm{H}}=10$. Another dust model is represented by the black dashed line, which is similar to the previous, but with a size range confined to small grains in the range $50 \leq a \leq 300 \AA$. The absorbed SED in Fig. A. 2 corresponds to $N_{20}^{\mathrm{H}}=3.4$. The last extinction considered is a model of the SMC extinction consisting of amorphous carbon grains with grain sizes comprised within the limits $50 \leq a \leq 1400$, as calculated by H07. The dust column assumed in Fig. A. 2 is $N_{20}^{\mathrm{H}}=10$.

The dust columns that we have assumed are arbitrary, but suffice to illustrate the contrasting behavior of the absorption in the far-UV that the various dust models produce. An inspection of Fig. A.2 shows that only nanodiamonds have the potential to reproduce the far-UV break observed in Ton 34 .

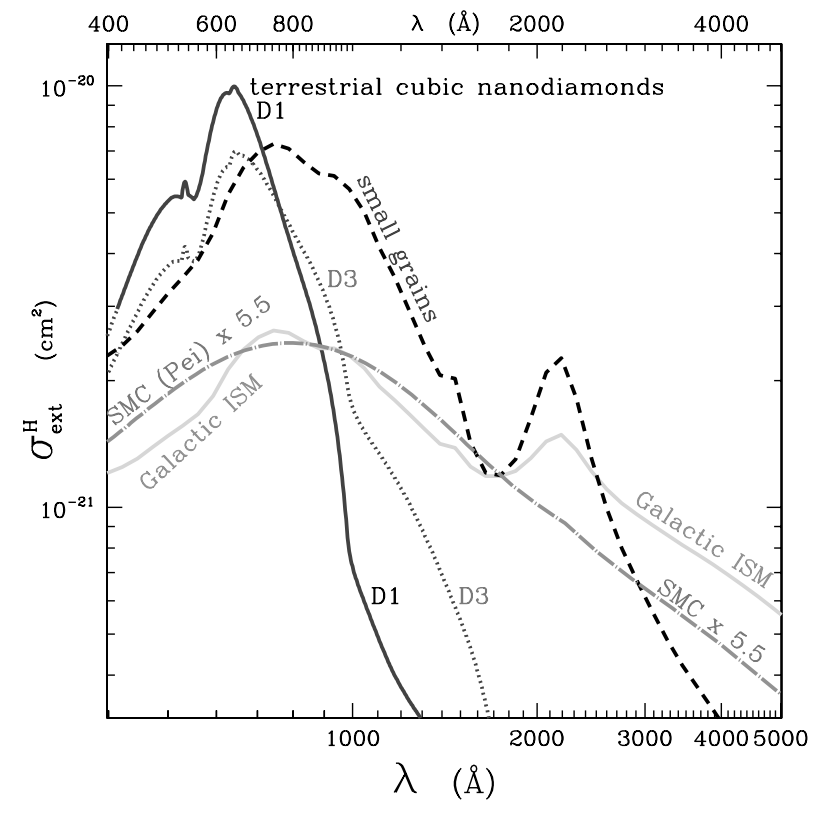

Fig. A.1. Comparison of the extinction cross sections from different dust models: black continuous line, (D1) nanodiamonds of sizes $\leq 25 \AA$ (see Sect. 3.1); dotted line, (D3) nanodiamonds of sizes extending up to $200 \AA$ (H07); continuous silver line, model by Martin \& Rouleau (1991) of the Galactic extinction consisting of graphite and silicates grains of sizes between 2500 and $50 \AA$; dashed line, extinction by graphite and silicate grains of small sizes $\leq 300 \AA$; long-dash-dotted line, the SMC dust extinction model of Pei (1992) multiplied by a factor 5.5 to facilitate comparison.

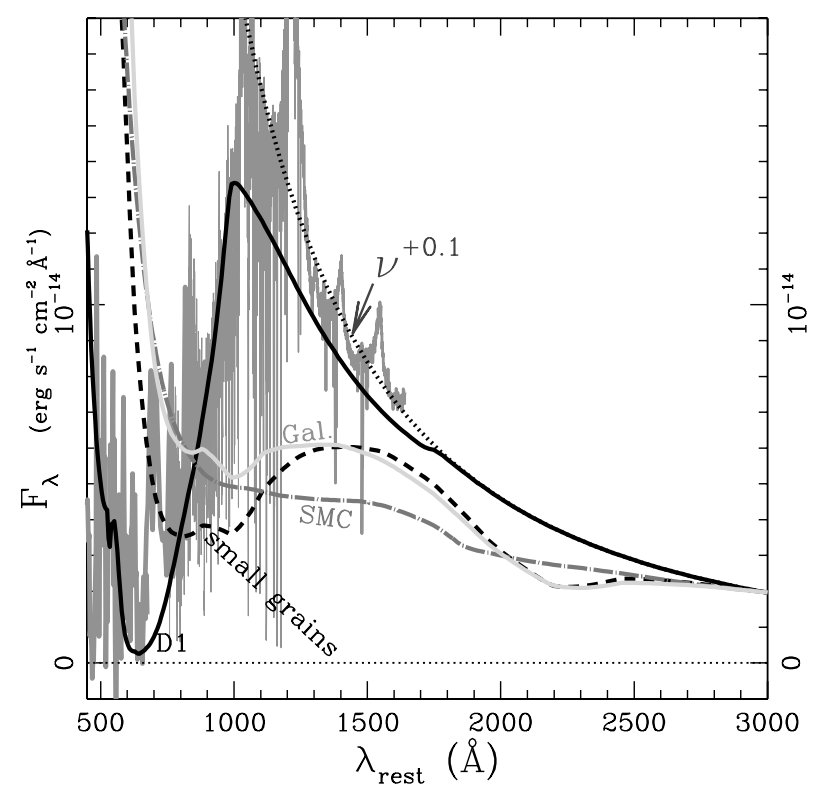

Fig. A.2. Comparison of the extinction that results from various dust models (cf. Fig. A.1). The assumed SED is a powerlaw of index +0.1 (dotted line). The Ton 34 spectrum is overlayed (thin grey line). To facilitate comparison, all curves shown were normalized to the same flux at $3000 \AA$. The curves are labeled as follows: black continuous line, nanodiamonds in small size regime (D1); continuous silver line, model by Martin \& Rouleau (1991) of the Galactic ISM extinction; dashed line, small graphite and silicate grains; long-dash-dotted line, SMC dust extinction (SMC dust model from H07).

Although dust in crystalline form is not common place in the Galaxy (Whittet 2002), this may not be the case in AGN. For 
instance, Roche et al. (2007) report the detection of a spectral structure near $11.2 \mu \mathrm{m}$ in NGC 3094, indicative of the possible presence of crystalline silicates. The presence of an UV radiation field might favor the formation of nanodiamonds through one of the following processes: UV annealing of carbonaceous grains (Nuth \& Allen 1992), nucleation in organic ice mixtures by UV photolysis (Kouchi et al. 2005) or chemical conversion of PAH clusters to nanodiamonds (Duley \& Grishko 2001). The absence of silicates could be explained by its significantly lower sublimation temperature. The resilience of small nanodiamonds led Rouan et al. (2004) to favor these as candidates to explain the IR emission from the 4 elongated nodules that they spatially resolve ( $K, L$ and $M$ bands) in the nucleus of NGC 1068.

\section{References}

Anderson, S. F., \& Margon, B. 1987, ApJ, 314, 111

Andersen, A. C. 1999, Ph.D. Thesis, University of Copenhaguen

Andersen, A. C., Jørgensen, U. G., Nicolaisen, F. M., Sørensen, P. G., \& Glejbol, K. 1998, A\&A, 330, 1080

Arav, N. 2004, AGN Physics with the Sloan Digital Sky Survey, ASP Conf. Ser., 311,213

Avni, Y., Worrall, D. M., \& Morgan, W. A. 1995, ApJ, 454, 673

Baker, J. C., \& Hunstead, R. W. 1995, ApJ, 452, L95

Baldwin, J., Ferland, G., Korista, K., \& Verner, D. 1995, ApJ, 455, L119

Binette, L., \& Krongold, Y. 2008, A\&A, 477, 413

Binette, L., Courvoisier, T. J.-L., \& Robinson, A. 1988, A\&A, 190, 29

Binette, L., Magris, G., Krongold, Y., et al. 2005, ApJ, 631, 661 (B05)

Binette, L., Haro-Corzo, S., Krongold, Y., \& Andersen, A. C. 2007, in Proc. of The nuclear region, host galaxy and environment of active galaxies, Huatulco, Méx., 18-20 April 2007, ed. E. Benítez, I. Crúz-Gonzalez, \& Y. Krongold, Rev. Mexicana Astron. Astrofis. Conf. Ser., in press, [arXiv:astro-ph/0706.2006]

Braatz, A., Ott, U., Henning, T., Jäger, C., \& Jeschke, G. 2000, Meteor. Planet. Sci., 35, 75

Cardelli, J. A., Clayton, G. C., \& Mathis, J. S. 1989, ApJ, 345, 245

Casebeer, D. A., Leighly, K. M., \& Baron, E. 2006, ApJ, 637, 157

Crenshaw, D. M., Kraemer, S. B., Bruhweiler, F. C., \& Ruiz, J. R. 2001, ApJ, 555,633

Crenshaw, D. M., Kraemer, S. B., \& George, I. M. 2003, ARA\&A, 41, 117

Czerny, B., Li, J., Loska, Z., \& Szczerba, R. 2004, MNRAS, 348, L54

Dhanda, N., Baldwin, J. A., Bentz, M. C., \& Osmer, P. S. 2007, ApJ, 658, 804

de Diego, J. A., Binette, L., Ogle, P., et al. 2007, A\&A, 467, L7

Draine, B. T., \& Lee, H. M. 1984, ApJ, 285, 89

Duley, W. W., \& Grishko, V. I. 2001, ApJ, 554, L209

Eastman, R. G., MacAlpine, G. M., \& Richstone, D. O. 1983, ApJ, 275, 53
Edwards, D. 1985, Cubic Carbon (Diamond), in Handbook of Optical Constants of Solids, ed. E. Palik (Orlando, Florida, USA: Academic Press Inc., Harcourt Brace Jovanovich), 665

Elvis, M. 2000, ApJ, 545, 63

Everett, J. E. 2005, ApJ, 631, 689

Gaskell, C. M., \& Benker, A. J. 2007, in press [arXiv:0711.1013]

Gaskell, C. M., Goosmann, R. W., Antonucci, R. R. J., \& Whysong, D. H. 2004, ApJ, 616, 147

Green, P. J., Schartel, N., Anderson, S. F., et al. 1995, ApJ, 450, 51

Hamann, F., \& Ferland, G. 1999, ARA\&A, 37, 487

Haro-Corzo, S. A. R., Binette, L., Krongold, Y., et al. 2007, ApJ, 662, 145 (H07)

Jones, A.P., \& d'Hendecourt, L.B. 2000, A\&A, 355, 1191

Konigl, A., \& Kartje, J. F. 1994, ApJ, 434, 446

Koratkar, A., \& Blaes, O. 1999, PASP, 111, 1

Korista, K., Baldwin, J., Ferland, G., \& Verner, D. 1997, ApJS, 108, 401 (KO97)

Kouchi, A., Nakano, H., Kimura, Y., \& Kaito, C. 2005, ApJ, 626, L129

Krongold, Y., Nicastro, F., Elvis, et al. 2007, ApJ, 659, 1022

Lanzetta, K. M., Turnshek, D. A., \& Sandoval, J. 1993, ApJS, 84, 109

Lawson, A. J., Turner, M. J. L., Williams, O. R., Stewart, G. C., \& Saxton, R. D. 1992, MNRAS, 259, 743

Li, A. 2007, in Proc. of The central engine of active galactic nuclei, in Xi'an, China, ed. L. C. Ho, J.-M. Wang, ASP Conf. Ser., 373, 561

Martin, P. G., \& Rouleau, F. 1991, in Proc. of Extreme Ultraviolet Astronomy (New-York: Pergamon), ed. R. F. Malina, \& S. Bowyer, 341

Mathis, J. S., Rumpl, W., \& Nordsieck, K. H. 1977, ApJ, 217, 425

Møller, P., \& Jakobsen, P. 1990, A\&A, 228, 299

Nuth, J. A., III, \& Allen, J. E., Jr. 1992, Ap\&SS, 196, 117

Pei, Y. C. 1992, ApJ, 395, 130

Pettini, M. 2006, Stellar Evolution at Low Metallicity: Mass Loss, Explosions, Cosmology, 353, 363

Reimers, D., Köhler, S., Hagen, H.-J., \& Wisotzki, L. 1998, Ultraviolet Astrophysics Beyond the IUE Final Archive, ESA SP-413, 579

Richards, G. T., Hall, P. B, Vanden, B. D. E., et al. 2003, AJ, 126, 1131

Roche, P. F., Packham, C., Aitken, D. K., \& Mason, R. E. 2007, MNRAS, 375, 99

Rouan, D., Lacombe, F., Gendron, E., et al. 2004, A\&A, 417, L1

Sargent, W. L. W., Boksenberg, A., \& Steidel, C. C. 1988, ApJS, 68, 539

Schlegel, D. J., Finkbeiner, D. P., \& Davis, M. 1998, ApJ, 500, 525

Shang, Z., Brotherton, M. S., Green, R. F., et al. 2005, ApJ, 619, 41

Telfer, R. C., Zheng, W., Kriss, G. A., \& Davidsen, A. F. 2002, ApJ, 565, 773 (TZ02)

Tripp, T. M., Bechtold, J., \& Green, R. F. 1994, ApJ, 433, 533

Vernet, J., Fosbury, R. A. E., Villar-Martín, M., et al. 2001, A\&A, 366, 7

Voges, W., Aschenbach, B., Boller, Th., et al. 1999, A\&A, 349, 389

Whittet, D. C. B. 2002, Dust in the galactic environment, second edition (Bristol: IOP)

Williams, O. R., Turner, M. J. L., Stewart, G. C., et al. 1992, ApJ, 389, 157

Yuan, W., Brinkmann, W., Siebert, J., \& Voges, W. 1998, A\&A, 330, 108

Zheng, W., Kriss, G. A., Telfer, R. C., Grimes, J. P., \& Davidsen, A. F. 1997, ApJ, 475, 469 